combination of haloperidol with promethazine for rapid tranquillisation may not be the most cost-effective or the most efficacious even when resources are poor.

Alexander et al used the Clinical Global Impression Scale to rate aggression and violence. We feel that use of more aggression-specific measures, such as the Overt Aggression Scale (Coccaro et al, 1991), which assesses different aspects of aggression and its severity, would have generated more specific results.

Alexander et al also showed that the combination injection produces sedation quicker than intramuscular lorazepam. However, this finding should be viewed with caution because the lorazepam group included more patients with mania, more patients with substance misuse or already on benzodiazepines (who could have developed tolerance to benzodiazepines) and more patients with marked or severe illness (which would necessitate a higher dose of medication to control aggression and violence). Together these factors might have contributed significantly to the results.

Alexander, J., Tharyan, P., Adams, C., et al (2004) Rapid tranquillisation of violent or agitated patients in a psychiatric emergency setting. Pragmatic randomised trial of intramuscular lorazepam $v$. haloperidol plus promethazine British Journal of Psychiatry, 185, 63-69.

CIMS (2004) CIMS - Updated Prescribers' Handbook, July (update3), pp. 203-236. Bangalore: Atmedica India.

Coccaro, E. F., Harvey, P. D., Kupsaw-Lawrence, E. et al (199I) Development of neuropharmacologically based behavioural assessments of impulsive aggressive behaviour. Journal of Neuropsychiatry and Clinical Neuroscience, 3, s44-s5l.

Hughes, D. H. \& Kleespies, P. M. (2003) Treating aggression in the psychiatric emergency service. Journal of Clinical Psychiatry, 64 (suppl. 4), 10-15.

McAllister-Williams, R. H. \& Nicol Ferrier, I. (2002) Rapid tranquillisation: time for a reappraisal of options for parenteral therapy. British Journal of Psychiatry, $\mathbf{1 8 0}$ 485-489.

S. Ranjan, P. S. Chandra National Institute of Mental Health and Neurosciences, Bangalore, India. E-mail: esarsingh@yahoo.co.in

Authors' reply: We thank Drs Ranjan and Chandra for their considered response to our article. Although we acknowledge variations in prescribing practice, we know of at least two other centres nearby (the Institute of Mental Health and the Government Hospital, Chennai) that use the haloperidol-promethazine combination for rapid tranquillisation; the monthly combined out-patient attendance of the three centres is also greater than 9000 .

Our wider survey of drug formularies, including the source of Ranjan and Chandra, and local pharmacies reveals that the price of injectable haloperidol $(5 \mathrm{mg} /$ ampoule) ranges between Rs 4.00 and Rs 5.50; that of promethazine $(50 \mathrm{mg} /$ ampoule) between Rs 3.00 and Rs 7.00 , and that of lorazepam ( $4 \mathrm{mg} / \mathrm{ampoule}$ ) between Rs 7.00 and Rs 15.00. We therefore reiterate our contention that the haloperidol-promethazine mix is cheaper than (even reduced doses of) haloperidol and lorazepam.

We agree that the Overt Aggression Scale would have generated more specific results. However, the outcomes for this pragmatic trial were not chosen to generate specific results; they were chosen by the doctors and nurses of the emergency rooms to be of clinical utility. From the reaction we have already had to this study these outcomes do seem acceptable and welcome to others.

We acknowledge that there were nine more people with mania, six more misusing substances and five more already on benzodiazepines in the lorazepam arm than in the comparison arm. There is no indication, however, that the integrity of the randomisation procedure was compromised, as such chance imbalances could occur in the absence of stratification. It is unlikely that these imbalances account for the findings, as the difference in the numbers of people 'clinically improved' between the two interventions at $15,30,60$ and $120 \mathrm{~min}$ were 31 , 25, 20 and 14, respectively, and in numbers 'asleep' 40, 47, 35 and 14.

Although recommended by important review articles and guidelines, we have found only four randomised studies in which a total of 80 people received the combination of haloperidol and lorazepam (Arana et al, 1986; Battaglia et al, 1997; Bieniek et al, 1998; Subramaney et al, 1998). None of these studies reports useful data on time to tranquillisation/sleeping; most report scale-derived data that are difficult to interpret clinically. For such limited data to direct practice at the two largest psychiatric centres in India, as well as many other places, would seem imprudent. The effects of haloperidol plus promethazine, we would still suggest, are better proven than other prevalent approaches. Recent influential guidelines in the UK have noted this and the sister study (TREC Collaborative Group, 2003) to be the only large trials of high methodological quality in this area (National Collaborating Centre for Nursing and Supportive Care et al, 2004).

Certainly the study and others like it need to be repeated so that the evidence upon which we treat people at this vulnerable time is robust. Practice on lesser evidence is surely unethical.

Arana, G. W., Ornsteen, M. L., Kanter, F., et al (1986) The use of benzodiazepines for psychotic disorders: a literature review and preliminary clinical findings. Psychopharmacology Bulletin, 22, 77-87.

Battaglia, J., Moss, S., Rush, J., et al (1997)

Haloperidol, lorazepam, or both for psychotic agitation? A multicenter, prospective, double-blind emergency department study. American Journal of Emergency Medicine, I5, 335-340.

Bieniek, S. A., Ownby, R. L., Penalver, A., et al (1998) A double-blind study of lorazepam versus the combination of haloperidol and lorazepam in managing agitation. Pharmacotherapy, 18, 57-62.

National Collaborating Centre for Nursing and Supportive Care (NCC-NSC), School of Health and Related Research, University of Sheffield (SchARR) \& Guideline Development Group (GDG) (2004) Clinical Practice Guidelines for the Short-term Management of Disturbed/Violent Behaviour in Adult Psychiatric In-patient Settings and Accident and Emergency Settings (Draft for 2nd Stage Consultation Period). http://www.nice.org.uk/pdf/DB_2ndcons_full. pd

Subramaney, U., Brook, S. \& Berk, M. (1998) A prospective randomised double-blind controlled study of the efficacy of lorazepam versus clothiapine in the control of acutely behaviourally disturbed patients. South African Medical Journal, 88, 307-310.

TREC Collaborative Group (2003) Rapid

transquillisation for agitated patients in emergency psychiatric rooms: a randomised trial of midazolam versus haloperidol plus promethazine. BMJ, 327 $708-713$.

J. Alexander, P.Tharyan Department of

Psychiatry, Christian Medical College, Vellore, India. E-mail: dralexander_in@yahoo.com

Clive Adams Cochrane Schizophrenia Group and Academic Unit of Psychiatry and Behavioural Sciences, University of Leeds, UK

Thomas John Department of Psychiatry,

Christian Medical College, Vellore, India

Carina Mol University of Ulm, Germany

Joncy Philip Department of Psychiatry, Christian Medical College, Vellore, India

\section{Limitations of rapid tranquillisation trial}

In their excellent paper Alexander et al (2004) systematically conducted a comparison trial of intramuscular lorazepam and haloperidol-promethazine in violent or agitated patients. The authors utilised a prospective follow-up design and used proper diagnostic assessment measures, thus taking care of most of the 
methodological pitfalls that have plagued research in this area.

However, a few concerns about the study persist. Ideally, there is a need for a viable placebo arm to compare the efficacy of both interventions. A comparison of a new agent with a drug previously shown to be active without a placebo comparator is uninterpretable unless one agent is superior to the others. Concluding that a drug is efficacious without a placebo comparator can lead to an incorrect assumption of efficacy if neither the investigational drug nor the active drug was, in that trial, any better than placebo would have been if included. Introducing a drug into therapeutic use on the basis of such a trial would expose patients to a compound with no greater benefit than placebo (Temple \& Ellenberg, 2000). A placebo is also important in the assessment of the safety profile, as it provides a base for determining which adverse events are truly related to the investigational drug. For these reasons, placebo-controlled trials are almost universally demanded by regulatory bodies to demonstrate efficacy for any pharmacological intervention.

The authors have not described any investigations carried out to exclude toxic states, epilepsy and other organic conditions. They failed to comment on vital parameters during and after administration of both interventions. They could have assessed the level of satisfaction of the treatment team with the intervention (Petrack et al, 1996). They could also have applied any scale for aggression, agitation, alertness and psychopathology (Battaglia et al, 1997).

Certain issues merit consideration before accepting the authors' conclusion. The better outcome of the haloperidolpromethazine group compared with the lorazepam group could be because the combination group had more patients with mania than the lorazepam group and the combination group had more moderately ill and less severely ill patients than the lorazepam group. In addition, details of additional medications were not mentioned. It remains possible that some improvement was due to additional medications in both groups.

The authors commented that 23 patients failed to sleep at all during the 4-h follow-up compared with only 8 in the combination group, which is difficult to understand from Table 2. There were some inconsistent findings in the paper: sleep outcome in the combination group at $120 \mathrm{~min}$ were $69 \%$ and $88 \%$ in Table 2 and Table 5, respectively. Similarly, there was a discrepancy in the number of patients in the combination group who were never tranquil (Results and Table 2).

Nevertheless, we feel that the authors have taken a useful step in this relatively neglected area. Further studies are required on the effectiveness of these interventions in the hope that better understanding can lead to better treatment of violent patients.

Alexander, J., Tharyan, P., Adams, C., et al (2004) Rapid tranquillisation of violent or agitated patients in a psychiatric emergency setting. Pragmatic randomised trial of intramuscular lorazepam v. haloperidol plus promethazine. British Journal of Psychiatry, 185, 63-69.

Battaglia, J., Moss, S., Rush, J., et al (1997)

Haloperidol, lorazepam, or both for psychotic agitation? A multicenter, prospective, double-blind, emergency department study. American Journal of Emergency Medicine, 15, 335-340.

Petrack, E. M., Marx, C. M. \& Wright, M. S. (1996) Intramuscular ketamine is superior to meperidine, promethazine, and chlorpromazine for paediatric emergency department sedation. Archives of Paediatric and Adolescent Medicine, 150, 676-681.

Temple, R. \& Ellenberg, S. (2000) Placebo-controlled trials and active control trials in the evaluation of new treatment. Part I: ethical and scientific issues. Annals of Internal Medicine, I33, 455-463.

O. P.Jhirwal, P. Kulhara, D. Basu Department of Psychiatry, Postgraduate Institute of Medical Education \& Research, Chandigarh-160012, India. E-mail: drjhirwalop@yahoo.co.in

Authors' reply We thank Dr Jhirwal et al for their interest in our pragmatic trial. In this reply we shall address only those issues that have not already been covered in response to earlier comments.

The first concern regarded the omission of a placebo arm. A placebo group was initially considered but abandoned as clinicians felt this was unethical, difficult to justify and likely to pose practical difficulties in implementation. This was a pragmatic trial and the design was driven by what questions clinicians wanted answered and what interventions they (and the institution's ethics committee) would permit. Moreover, systematic reviews reveal no evidence that placebo interventions in general have clinically important effects, and the role of placebos in clinical trials, apart from helping to minimise bias, is questionable (Hróbjartsson \& Gøtzsche, 2004). Our pragmatic trial utilised adequate allocation concealment and masking of primary outcome assessors, two crucial features of trial design that significantly affect the internal validity of a randomised controlled trial (Jüni et al, 2001).

Those with toxic states, epilepsy or other organic conditions were invariably excluded from the study as treating clinicians were uncomfortable about their inclusion in a randomised trial with sedative agents. Investigation results are rarely available before the intervention is instituted for violent patients under normal conditions of clinical practice.

All those subjected to tranquillisation received standard levels of care that included monitoring of vital signs and intensive nursing support. Any adverse events with regard to autonomic instability were promptly reported. Only two patients on lorazepam reported any adverse events and this is described in the discussion (paragraph 4, page 65; a printing error in Table 2 ascribes this to the combination group instead of to lorazepam).

Table 2 records that equal numbers in both groups were given additional medication (always a single dose of $100 \mathrm{mg}$ chlorpromazine) and this contradicts the speculation that differences in additional medication could have influenced improvement in favour of any particular group. The proportions that failed to sleep or were never tranquil reported in paragraph 1 on page 65 are correct. Table 2 reports the numbers who were tranquil/asleep and asleep at the times when outcomes were recorded. People who were tranquil or asleep at one assessment did not invariably remain so at other assessments, hence explaining the apparent discrepancy. We acknowledge the error in Table 5 where the proportion asleep at $120 \mathrm{~min}$ should be $69 \%$ and not $88 \%$ for TREC-India.

We hope the interest aroused by this paper will prompt greater use of trials of pragmatic design, free of industry sponsorship and aimed at answering clinical questions of relevance to real world clinical practice.

Hróbjartsson, A. \& Gøtzsche, P. C. (2004) Placebo interventions for all clinical conditions. Cochrane Database of Systematic Reviews, issue 2 (article no.: CD003974.pub2. DOI: 10.1002/14651858.CD003974. pub2). Chichester: Wiley Interscience.

Jüni, P., Altman, D. G. \& Egger, M. (200I) Systematic reviews in health care: Assessing the quality of controlled clinical trials. BMI, 323, 42-46.

J. Alexander, P.Tharyan Department of Psychiatry, Christian Medical College, Vellore, India. E-mail: dralexander_in@yahoo.com 